La diversidad de la securización urbana: dimensiones, actores y retos

\title{
Usos políticos del concepto de seguridad humana: securitización de la violación de derechos humanos y del subdesarrollo en el escenario internacional ${ }^{*}$
}

Political Uses of the Concept of Human Security: Securitization of the Violation of Human Rights and Underdevelopment in the International Scenario

Usos politicos do conceito de segurança humana: securitização da violação de direitos humanos e do subdesenvolvimento no cenário internacional

Julián Andrés Muñoz Tejada**

Recibido: 10 de noviembre de 2017

Aprobado: 11 de abril de 2018

Doi: http://dx.doi.org/10.12804/revistas.urosario.edu.co/territorios/a.6232

Para citar este artículo:

Muñoz, T. J. A. (2018). Usos políticos del concepto de seguridad humana: securitización de la violación de derechos humanos y del subdesarrollo en el escenario internacional. Territorios (39), 21-46. Doi: http:// dx.doi.org/10.12804/revistas.urosario.edu.co/territorios/a.6232

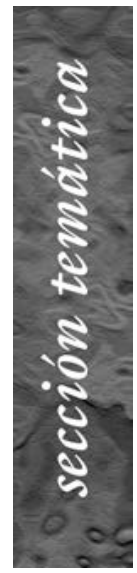

* Desarrollo parcial de la investigación que realiza el autor para optar por el titulo de Doctor en Ciencias Sociales en la Universidad de Antioquia. El título de la investigación es "Usos políticos del concepto de seguridad humana. El caso del Observatorio de Seguridad Humana de Medellín —OSHM-2008-2015”. El autor agradece los comentarios de quienes evaluaron este articulo.

** Abogado, Especialista en derecho penal, Magister en Ciencia Politica y candidato a doctor en Ciencias Sociales. Profesorvinculado a la Facultad de Derecho y Ciencias Politicas de la Universidad de Antioquia, donde ha impartido cursos en suspregrados de Ciencia Politica y Derecho. Miembro del Grupo sobre Conflictos, Violencias y Seguridad 
Palabras clave

Seguridad humana, securitización, responsabilidad de proteger, subdesarrollo, derechos humanos.

Keywords

Human security, securitization, responsibility to protect, underdevelopment, human rights

Palavras-chave

Segurança bumana, securitização, responsabilidade de proteger, subdesenvolvimento, direitos humanos

territarias 39

\section{RESUMEN}

En este artículo se hace una revisión documental sobre algunos usos políticos del concepto 'seguridad humana' en el escenario internacional. El artículo muestra cómo esos usos en el escenario internacional se concretaron en procesos de securitización en torno a la violación de derechos humanos y el subdesarrollo en algunos contextos. Se explora cómo se formaron discursos de seguridad humana claramente diferenciables, lo que confirma el carácter equívoco de los conceptos en la política y cómo, si se estudian los usos que determinados actores les dieron en contextos específicos, se pueden conocer sus significados.

\section{ABSTRACT}

In this article, a documentary review is made about some political uses of the concept of human security in the international scenario. The article shows how these uses in the international scenario were concretized in securitization processes around the violation of human rights and of underdevelopment in some contexts. It explores how clearly differentiable human security discourses were formed, which confirms the equivocal nature of the concepts in politics, and how to study the uses that specific actors gave them in specific contexts can know their meanings.

\section{RESUMO}

Neste artigo se faz uma revisão documental sobre alguns usos políticos do conceito 'segurança humana' no cenário internacional. O artigo mostra como esses usos no cenário internacional concretizaram-se em processos de securitização em torno à violação de direitos humanos e o subdesenvolvimento em alguns contextos. Explora-se como se formaram discursos de segurança humana claramente diferenciáveis, o que confirma o carácter equívoco dos conceitos na política e como se se estudam os usos que determinados atores lhes deram em contextos específicos se podem conhecer seus significados. 


\section{Introducción}

En este artículo se exploran algunos usos políticos del concepto de seguridad humana en el escenario internacional. Se deriva de una investigación documental que recurrió al horizonte de intelección que posibilita la Teoría del Discurso de autores como Laclau, Wittgenstein y Foucault. Concretamente, se recurre a algunos elementos del Análisis Político del Discurso (APD), una perspectiva de análisis que se desarrolla en el marco del "giro lingüístico" de las ciencias sociales.

El APD expresa un horizonte de intelección de la realidad, antes que un conjunto fijo de teorías o métodos, sin que ello implique que esté desprovisto de categorías teóricas o que prescinda de estrategias metodológicas (Navarrete, 2009). La analítica del discurso en esta perspectiva sugiere una:

[...] potente amalgama conceptual condensada en la noción de discurso [que] atañe a toda la producción del hombre, ya que la significación atraviesa el quehacer social de un extremo a otro y no se puede acceder a dicho quehacer sin poner en movimiento ni atravesar esa textura (Saur, 2017, p. 6).

Es importante aclarar que aquí no se dará cuenta de la emergencia y de los usos políticos de la seguridad humana en todos los escenarios posibles en el nivel de las relaciones internacionales, lo que sería francamente irrealizable. Solo se abordarán dos de los usos políticos del concepto de 'seguridad humana' en el ámbito internacional: cuando es usado como fundamento de la responsabilidad de proteger para securitizar las violaciones de derechos humanos en algunos contextos y como fundamento de algunos programas de Desarrollo para securitizar el subdesarrollo de algunos países.

Lejos de suponer un cuerpo de enunciados y principios de acción coherentes —política y analíticamente-, la seguridad humana se ha movido entre: (i) la crítica a los modelos militaristas herederos de la Doctrina de la Seguridad Nacional por la doctrina de la seguridad nacional y (ii) una nueva ortodoxia sobre esta materia, cuando actores poderosos lo han usado. Se configuran, por lo tanto, dos discursos de seguridad humana: uno que critica visiones militaristas sobre seguridad y otro que las reproduce.

Un discurso puede entenderse como el resultado de un proceso de significación; no es una totalidad cerrada, "sino abierta, incompleta y precaria que involucra el carácter relacional y diferencial de los elementos" (Navarrete, 2009, p. 146). Por ello, un discurso de seguridad humana, antes que un conjunto cerrado y fijo de enunciados y prácticas sobre seguridad, derechos humanos y desarrollo humano, es una manera de pensar esas cuestiones que permanentemente se están actualizando y renovando. Un discurso de seguridad humana, en suma, se refiere al resultado de un proceso de significación, que incluye unas condiciones de emergencia y unos usos políticos en concreto, que cabe analizar si
Humana de la misma universidad. Correo electrónico: julian.munozt@udea. edu.co. ORCID: https://orcid.org/0000-0003-18519196

territarios 39

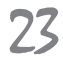


${ }^{1}$ Se asume aquí que la equivocidad es un rasgo de todo el lenguaje, incluido el lenguaje político, (Gallardo, 2014), y por ello resulta desproporcionado suponer que esta es una característica especialmente visible en el caso la seguridad humana, como si los Derechos Humanos, la dignidad humana, la libertad o la seguridad si fueran univocos en sus significados. Si se exploran los usos concretos de un concepto se puede comprender su significado a partir de la manera como determinados actores se apropiaron de él.

\section{tersitarias 39}

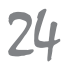

se quiere entender el significado concreto de un concepto.

Por ello, como afirma Wittgenstein (1999, 2011), conociendo los usos de un signo, y podría agregarse aquí de un concepto, es posible entender su significado. En el caso del concepto de seguridad humana, al explorar cómo se usó por algunos actores en el escenario internacional, se puede entender cómo se construyeron algunos de los significados de este concepto que aparece en la década de los noventa, vinculado con los otros como desarrollo humano y derechos humanos.

Un uso político remite a la manera como determinados actores aprovechan la vaguedad e imprecisión de los conceptos ${ }^{1}$; a jugar el juego (De Certeau, 2007), por ejemplo, de "los derechos humanos", del “desarrollo humano" y, claro, de la "seguridad humana". Todos ellos serían significantes vacíos, como diría Laclau (1996). Su significado siempre será contingente y posible gracias al contexto en el que se usan y los usos concretos que hacen de ellos algunos actores.

Se forman, en este sentido, al menos dos discursos de seguridad humana en el escenario internacional. El primero de ellos plantea una visión que criticaba los discursos militaristas que hasta 1994 se habían difundido - en especial el de la Doctrina de la Seguridad Nacional- y suponía que lo inseguro debía entenderse desde una perspectiva mucho más amplia, que incluyera distintas dimensiones para pensar los hechos o situaciones que podrían constituir amenazas a la seguridad de las personas.

Se tiene también un segundo discurso de seguridad humana, que en el escenario internacional se desliga del primero y plantea que se debe intervenir en algunos países - a los que se define como generadores de inseguridad-, para garantizar la seguridad a nivel global. Los lugares donde se realizan esas intervenciones se caracterizan por padecer conflictos armados, no tener unos niveles óptimos de desarrollo o por ambas cosas. Dicho de otra manera, para evitar que esos países contagien su inseguridad a otros, deben por ello ser intervenidos.

Según este último discurso, en el escenario internacional, la seguridad humana funciona como un dispositivo de control que posibilita la administración de los desórdenes y la gestión de riesgos derivados de la inseguridad que algunos Estados débiles, fallidos o subdesarrollados representan para la seguridad de otros asumidos como fuertes o desarrollados, que a su vez hablan en nombre de la seguridad global.

Al hablar de securitización, se alude a procesos por medio de los cuales un asunto se construye como problema de seguridad. Cuando un asunto es definido como un problema de seguridad - valga decir, cuando es securitizado-, supone la existencia de una amenaza a un objeto referente - un interés, un bien jurídico, etc. - asumido como importante dentro de una comunidad y la necesidad de generar respuestas a dicha amenaza (Buzan, Wæver \& de Wilde, 1999). 
Como diversos autores han planteado, en principio, habría una serie de desventajas asociadas con la idea de ampliar lo que se puede entender como problemas de seguridad. Una agenda demasiado amplia de seguridad, sin pensarlo, la eleva a la categoría de bien universal, lo que puede propiciar que los Estados privilegien los mecanismos de emergencia -incluso de tipo violento - para conjurar aquellos asuntos definidos como problemas de seguridad (Buzan, Wrever \& de Wilde, 1999).

Por otra parte, la securitización de algunos asuntos pone de presente que la definición y gestión de un problema de seguridad no dependa exclusivamente de condiciones objetivas relativas a las amenazas (Balzacq \& Guzzini, 2015). Lo importante no es tanto la materialidad de la amenaza, sino quién y por qué ahora decide construir un problema. Por ello, como plantea Balzacq (2011), cuando se estudian procesos de securitización se debe cuestionar la naturalidad de los problemas de seguridad y preguntar cómo se llegó a nombrar una cuestión como problema de seguridad.

La estructura del artículo es la siguiente: un primer apartado presenta algunas condiciones de contexto que posibilitaron la emergencia del concepto de seguridad humana. En la segunda parte se analizan dos usos políticos del concepto seguridad humana y por qué pueden leerse como procesos de securitización. Por último, se presentan algunos apuntes finales a modo de conclusión.

\section{Emergencia del concepto de seguridad humana}

La literatura sobre seguridad humana, en general, identifica dos posibles fuentes de inseguridad: el miedo y la necesidad, según lo cual se habla de dos concepciones de la seguridad humana, una restringida (freedom from fear), cuyo principal promotor a nivel internacional es el Estado de Canadá - seguido por otros Estados como Noruega y Suecia-, y otra amplia (freedom from want), usualmente vinculada con el Estado japonés (Morillas, 2007; Pérez de Armiño, 2013).

La primera reduce la inseguridad a la violencia física que pueden sufrir las personas, o a las muertes causadas en el marco de conflictos armados, así el Estado no sea una de las partes en la contienda, como ha mostrado el Human Security Centre, financiado por el gobierno canadiense; la segunda, por su parte, identifica todo un conjunto de nuevas dimensiones desde las cuales podría afectarse la seguridad de las personas.

En esta última concepción — promovida por el gobierno japonés-, se contempla un nuevo y ampliado catálogo de fuentes desde las que se podría afectar la seguridad de los seres humanos. La seguridad humana propone una manera de pensar la seguridad de los seres humanos como algo complejo que se puede afectar desde distintas dimensiones, todas ellas interrelacionadas: económica, alimentaria, sanitaria, medioambiental, personal, comunitaria, política e incluso de género. territarios 39

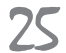


${ }^{2}$ La seguridad de los seres humanos es un asunto, pues, que concierne a la comunidad internacionaly no solo a los Estados, por lo cual la soberanía - que desde los tiempos de la Paz de Westfalia era asumida como un atributo absoluto de los Estados-, empieza a relativizarse, pues si un Estado no protege los derechos de sus ciudadanos, incluso podría ser intervenido por fuerzas extranjeras (MacFarlane \& Foong Khong, 2006)

${ }^{3}$ Nótese cómo, dentro de los paises mencionados no hay uno solo de aquellos a los que se conoce como desarrollados, sino solo los considerados como Estados débiles, subdesarrollados o fallidos, a pesar de que la seguridad humana evoca valores universales o comunes a todos (Thede, 2008).

\section{territarias 39} 26
Particularmente, esta última dimensión es todavía objeto de polémica, pues desde los análisis feministas se afirma que la seguridad humana todavía no ha incorporado una dimensión de género que complemente las otras 7 (Mendia \& Saillard, 2013).

Esta ampliación del campo discursivo de la seguridad sugirió que habría cambios importantes en la manera como se pensaba la seguridad internacional. Por ejemplo, Magallón apuntó que "la introducción del concepto de seguridad humana en el Informe sobre Desarrollo Humano del PNUD, en 1994 fue una pequeña revolución copernicana" (Magallón, 2007, p. 1). Dicho aserto se funda en la idea de que la seguridad humana permitiría ampliar el conjunto de problemas y dimensiones desde las cuales podría pensarse la seguridad.

No es que hayan desaparecido las viejas preocupaciones de los Estados de que se afecte su soberanía - aunque esta sea replanteada y ya no sea un absoluto $-^{2}$ y se entienda que los problemas de seguridad ya no se refieran a las instituciones estatales. Lo que ocurre es que, desde los años noventa, las discusiones sobre la seguridad se ampliaron y empezaron a contemplar asuntos que implicaban algo más que las amenazas al Estado y sus instituciones. Con la seguridad humana se empiezan a tener en cuenta como problemas de seguridad asuntos relacionados con la economía, el medio ambiente, la salud, el género, la cultura, entre otros (MacFarlane \& Foong Khong, 2006).

La ampliación de las fronteras de lo que puede ser asumido como un problema de seguridad, y sus complejas relaciones con nociones como derechos humanos y desarrollo humano, constituyen algunos de los aspectos con que más se cuestiona al concepto de seguridad humana, pues, en su afán de extender el ámbito de las amenazas y los objetos referentes, complejiza enormemente definir lo que es propio de la seguridad y diferenciarlo del desarrollo humano y los derechos humanos.

Con todo, y que pese a su vaguedad e imprecisión, para algunos defensores del concepto, la seguridad humana no promueve una revisión permanente de todo, sino que prioriza aquellos contextos en los que se identifiquen focos especialmente graves de inseguridad que por lo general coinciden con países de especial conflictividad, tales como Afganistán, Palestina, Bangladesh, Estonia, Macedonia, Sierra Leona, Timor Oriental, (Gasper, 2015) o aquellos de la región del Cáucaso Norte (Ferreira, 2013) ${ }^{3}$.

El concepto de seguridad humana propone, inicialmente, al finalizar la Guerra Fría, romper con las visiones más militaristas heredadas de la Doctrina de la Seguridad Nacional. Plantea que las principales amenazas a la seguridad no se ciernen sobre los Estados, sino sobre las personas y que la seguridad se puede afectar desde múltiples dimensiones, todas ellas interconectadas.

En sus inicios, la seguridad humana, fue un concepto ligado a las nociones de desarrollo humano y derechos humanos (Programa de las Naciones Unidas para el Desarrollo —PNUD—, 1994). Surge a mediados de los años noventa como una 
respuesta a la orientación neoliberal de los mercados y en medio de unas condiciones históricas que lo vinculan al fenómeno de la globalización (Roznai, 2014).

Como se mostrará en otra publicación, y se menciona en el apartado final de este artículo, además de los usos internacionales aquí expuestos, en Colombia también se usó políticamente este concepto. Se pueden enunciar, a modo de ejemplo:

(i) Los programas que financió la Unidad de Seguridad Humana de las Naciones Unidas en el Departamento de Cundinamarca entre los años 2004 y 2005 , y en el municipio de Soacha entre los años 2010 y 2013;

(ii) El Plan de Desarrollo del Municipio de Ibagué, "Camino a la Seguridad Humana", durante el periodo 20122015;

(iii) Uno de los fundamentos de los Acuerdos de Paz suscritos entre el Gobierno Nacional y la guerrilla de las Farc en el 2015;

(iv) Uno de los fundamentos planteados por un grupo armado ilegal (la Oficina de Envigado) para entablar negociaciones con el Estado;

(v) Un discurso de seguridad humana "desde abajo" constituido por el Observatorio de Seguridad Humana de Medellín (OSHM) entre los años 20082016.

No se trata de incomprensiones o usos equívocos del concepto de seguridad humana en ninguno de estos casos, sino de apropiaciones concretas que cada uno de los actores señalados hizo del concepto en mención. Cada uno de ellos se "valió de él" para fines diversos; por ejemplo, el osHM lo hizo para criticar el discurso militarista instaurado en Colombia tras la Política de Defensa y Seguridad Democrática, y constituir en su lugar una forma alterna para re-pensar la seguridad ${ }^{4}$.

Retomando, tras el fin de la Guerra Fría, nuevos desafíos en términos de seguridad, asociados con las dinámicas y retos que empezaba a plantear la globalización, empezaron a visibilizarse. Fenómenos como el cambio climático, los debates sobre el desarrollo en un contexto de liberalización de los mercados o las "nuevas guerras", entre otras cuestiones, cambiarían las prioridades de las agendas de seguridad en el escenario internacional, donde las distinciones entre el interior y el exterior de los Estados cada vez se desdibujan de manera más pronunciada.

El contexto de emergencia en el que se forma la seguridad humana se inscribe dentro del periodo de la pos-Guerra Fría, caracterizado ya no por la bipolaridad, sino por el dominio ${ }^{5}$ de Estados Unidos (Kamal, 2007), no solo en términos militares, sino también económicos, políticos e incluso culturales. Aunque ha habido desafíos a dicho orden, este, en general, se ha mantenido, sobre todo a partir de la Guerra Internacional contra el Terrorismo que declara el expresidente George W. Bush, en virtud de la cual movilizó tropas para invadir Afganistán e Irak, por su presunta relación con redes terroristas.
${ }^{4}$ En el sitio web del OSHM (bttp://www.repensandolaseguridad.org) se pueden encontrar las publicaciones e iniciativas que ha liderado o acompañado este observatorio.

${ }^{5}$ Hay un debate en relación con la hegemonía de los Estados Unidos, pues Michael $y$ Marshall (2007) consideran que Estados Unidos sí logró constituir una begemonía (Kamal, 2007). Para este último, Estados Unidos es un "Imperio sin hegemonia”, pues una relación hegemónica no pretende borrar las diferencias - como si ha procurado bacerlo los Estados Unidos en su guerra contra el terrorismo-, sino reconocerlas.

territarias 39

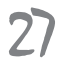


- Se entiende por campo discursivo de la seguridad, parafraseando a Bourdien (1990), a aquel escenario donde se define el significado de la seguridad. Se trata de un escenario caracterizado por tensiones entre "intereses irreductibles" $y$ actores que disputan ese significado.

${ }^{7}$ Aunque hay Estados, como Estados Unidos, con toda una tradición de intervenciones - militar, por medio de programas de desarrollo o ambas - mucho más 'rica' y elocuente que Canadá o Japón, aqui se enfatiza en estos, pues sus agencias son las que han desarrollado y aplicado el concepto de seguridad humana de manera más consistente.

\section{tersitarias 39}

Así, desde finales de los años ochenta del siglo pasado, se empezó a proponer el ensanchamiento del campo discursivo de la seguridad al sugerir que las fuentes de inseguridad no se podían identificar únicamente en las amenazas militares al Estado; desde ese momento también se podían localizar otras amenazas de tipo económico, social o medioambiental. La referida ampliación del campo discursivo de la seguridad ${ }^{6}$ permitió comprender que no solo el Estado puede ver amenazada su seguridad, sino también los individuos (Pérez de Armiño, 2007).

Llama la atención, sin embargo, que un concepto como el de seguridad humana - y junto a él el de desarrollo humanohaya sido pensado inicialmente como discurso crítico, de la mano de autores que no hacían parte del mainstream de los estudios de seguridad o desarrollo a nivel mundial, como el hindú Amartya Sen y el pakistaní Mahbub Ul Haq (Acharya, 2016).

Esto evidencia, de nuevo, el carácter instrumental de los conceptos, o cómo se les puede usar incluso para fines que abiertamente niegan los propósitos para los cuales fueron formulados inicialmente. Según se muestra en este artículo, fue eso lo que ocurrió cuando actores en el escenario internacional usaron el concepto de seguridad humana, principalmente, para mantener relaciones de sujeción de unos Estados sobre otros bajo el pretexto de fomentar el desarrollo o de proteger los derechos humanos de sus poblaciones.

El contenido crítico del concepto de seguridad humana parece haberse opacado cuando países poderosos o las mismas $\mathrm{Na}-$ ciones Unidas lo aplicaron; o, también, cuando algunos Estados —como Canadá, Japón y Noruega - lo usaron dentro de sus agendas de política exterior. Es como si se hubiera operado un tránsito de la heterodoxia que caracterizó este concepto en sus inicios cuando fue formulado por autores como Mahbub Ul Haq o el mismo Sen, a una nueva ortodoxia de las corrientes principales $^{7}$, cuando Estados poderosos lo usan y constituyen sentidos donde la crítica a los discursos militaristas heredados de los tiempos de la Guerra Fría ya no aparece, y sobre todo donde los vínculos entre el concepto de seguridad humana y los derechos humanos cada vez son más tenues.

De esta manera, el referido tránsito se explica por la manera como determinados actores en el escenario internacional se valieron del concepto - sobre todo en su acepción restringida de libertad respecto del miedo- para continuar con visiones que poco o nada significaron en términos de ruptura con la manera como se leía la seguriad en los tiempos de la Guerra Fría como un asunto que escencialmente concernía a los Estados y a su protección frente a amenazas tanto externas como internas.

Por ejemplo, el exministro de relaciones exteriores canadiense, Lloyd Axworthy, uno de los grandes promotores de la seguridad humana, ha alejado el concepto de sus orígenes - anclados al desarrollo humano (Bajpai, 2004) - para enfocarlo en aspectos que tradicionalmente han sido entendidos como propios de la seguridad - minas antipersona, Corte Penal 
Internacional e intervenciones humanitarias- (Acharya, 2016).

De esta manera, aunque la seguridad humana aparece ligada a los conceptos de desarrollo humano y derechos humanos, ha posibilitado toda una pluralidad de usos políticos (significados), que no necesariamente corresponden con la idea emancipadora $^{8}$, ligada a los derechos humanos y al desarrollo humano que inspiró esta forma de pensar la seguridad (Christie, 2010).

En el escenario internacional, la promoción de la seguridad humana ha contado con el apoyo destacado de los gobiernos de Canadá y Japón. Canadá, por ejemplo, cuando era miembro del Consejo de Seguridad de la ONU apoyó los bombardeos sobre Kosovo en 1999 (Rodríguez 2005) e hizo parte de la coalición de Estados que en el marco de la "Guerra internacional contra el terrorismo" intervino militarmente Afganistán en 2001, justo cuando se publica el informe "La Responsabilidad de Proteger" "que reformula y relativiza la soberanía estatal" (Pérez de Armiño, 2007 , p. 72) y ofrece justificaciones para dichas intervenciones.

En el caso de Japón, en 1998, adopta una política exterior que estaba enfocada en la promoción del empoderamiento y la emancipación de comunidades para reducir la violencia estructural, a partir de iniciativas de protección a cargo de los Estados, algunas ONG y entidades internacionales encargadas (Hynek, 2012). La aplicación de la seguridad humana en la política exterior japonesa ha estado ligada a iniciativas como la Comisión de
Seguridad Humana de Japón, creada en el 2001 para conceptualizarla, y la creación de la Unidad de Seguridad Humana y su Fondo Fiduciario, del cual Japón es el principal donante.

El gobierno japonés ha entendido con el concepto de seguridad humana que, si se protege a las personas que viven en contextos especialmente conflictivos, ello le permitiría protegerse en lo que supone un ejercicio coherente con su tradición política y con los nuevos desafíos que representa un mundo globalizado (García 2007). Ahora, incluso esta visión de la seguridad humana ha sido objeto de críticas, principalmente, por la idea universalista que late en ella de que hay algo "humano" común para todos los lugares y sujetos.

Como muestra Duffield (citado por Schering \& Wood, 2011), dicho universalismo ha estado atravesado por la guerra internacional contra el terrorismo, en la que no se distinguen especificidades contextuales de los conflictos en el mundo; se asume, en cambio, que en todos ellos la amenaza a la seguridad global es similar y que en todos está asociada con violaciones de derechos humanos y con problemas de subdesarrollo. La seguridad humana, en suma, es un concepto ligado al desarrollo humano y a los derechos humanos. Cuando se quiso con él ampliar la discusión sobre la seguridad y referirla a otras amenazas y objetos referentes ${ }^{9}$, posibilitó toda una pluralidad de significados (usos), que no necesariamente corresponden con la idea emancipadora que lo inspiró inicialmente (Christie, 2010).
${ }^{8}$ Se alude puntualmente a las tensiones que se pretendia superar entre los conceptos de libertad y seguridad heredados del pensamiento politico moderno, suelen presentarse de manera más o menos antitética -en especial a partir de obras como la de Hobbes-. Si se supone que la seguridad humana se refiere, sobre todo, a la posibilidad de ejercer libertades - frente al miedo y la necesidad-, no babria una oposición real entre libertady seguridad sino una relación de complementariedad.

${ }^{9}$ Las "amenazas" y los "objetos referentes” se entienden, según Buzan, De Wilde, y Wever (1999), como elementos vinculados con procesos de securitización, es decir, procesos en virtud de los que se definen los problemas de seguridad.

territarias 39

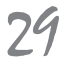


${ }^{10}$ El presente escrito se enfoca en la justificación de tipo estratégico, pues es la que refleja de manera mucho más clara cómo actores en el escenario internacional usaron políticamente el concepto de seguridad humana o, cómo se ha planteado, se "valieron de él”. Por ello, los otros dos tipos de justificación - moral y juridica- no serán tenidos en cuenta en esta oportunidad.

\section{territarios 39}

\section{Usos políticos del concepto de seguridad humana en el escenario internacional}

En los usos políticos de la seguridad humana que aquí se examinan, se pueden advertir dos procesos de securitización. En el primero de ellos, la seguridad humana se usa como fundamento de una doctrina de intervención de unos Estados en otros, cuando se producen afectaciones graves a los derechos humanos de quienes habitan sus territorios, con lo cual se construye como problemas de seguridad a las violaciones de derechos humanos; y en el segundo, se fomenta el desarrollo de algunas regiones para evitar que sus escasos niveles de desarrollo se traduzcan en problemas de seguridad que se podrían contagiar a otros países, con lo que se construye como problema de seguridad al subdesarrollo.

\subsection{La seguridad humana y la responsabilidad de proteger}

\subsubsection{La Responsabilidad de proteger}

Hay algunos antecedentes de la responsabilidad de proteger en los años noventa. Por ejemplo, los casos de Somalia, Rwanda, Srebrenica y Kosovo (Zabaleta 2014); las Resoluciones del Consejo de Seguridad de las Naciones Unidas 1265 de 1999 y 1296 de 2000, que apuntaron a la atenuación de la soberanía de los Estados en el contexto de misiones de paz para proteger a los civiles (Macfarlane 2004); o con la alusión a la "soberanía como responsabilidad", según la cual, como planteó el exdelgado de las Naciones Unidas para el desplazamiento de personas, Francis Deng (citado por Acharya, 2016), el Estado tiene la obligación de cuidar a sus ciudadanos, y, si necesita ayuda, debe pedirla a las organizaciones internacionales, pues si no lo hace y su pueblo sufre, el mundo no podría quedarse impasible y debería intervenir.

Se trata de una obligación de intervenir que reviste una triple justificación: moral, jurídica y estratégica ${ }^{10}$. La primera cuestión se refiere a una preocupación solidaria por los seres humanos; la segunda, a las obligaciones contraídas por los Estados de promover y respetar los derechos humanos en virtud de los Artículos 55 y 56 de la Carta de las Naciones Unidas; y la tercera alude a razones de autoprotección de los Estados ya que, por ejemplo, los europeos no pueden estar seguros mientras otras personas en el mundo vean afectada su seguridad de manera severa (Grupo de Estudios sobre las Capacidades de Europa, 2007).

El Informe de la Comisión Internacional sobre Intervención y Soberanía, cuya comisión conformó el gobierno canadiense en septiembre de 2000 (Comisión Internacional sobre Intervención y Soberanía de los Estados - CIISE- 2001), plantea que la responsabilidad de proteger se empieza a concebir como una forma de pensar para repensar el concepto de soberanía.

Como se indica en ese informe, el contexto de la pos-Guerra Fría en muchos países introdujo "nuevas cuestiones de seguridad" asociadas con "la democratización, 
los derechos humanos y la gobernanza. Pero en muchos otros trajo consigo guerras internas o conflictos civiles, casi siempre con terribles repercusiones políticas y humanitarias" (CIISE, 2001, pp. 4-5).

La indivisibilidad - $\mathrm{O}$ interconexión de los problemas - de seguridad en el nivel internacional es uno de los motivos por los que unos países destinan parte de sus recursos para asistir a otros con especial conflictividad. La responsabilidad de proteger es una doctrina sobre la intervención, que puede ser militar — aunque también puede incluir otro tipo de sanciones como las políticas, económicas o diplomáticas(CIISE, 2001).

En el contexto de la guerra internacional contra el terrorismo a partir de los ataques del 11-S, desatada por el gobierno estadounidense y que se traduce en las invasiones a Afganistán - en el 2001-e Irak —en el 2003-, en 2004 el Grupo de Alto Nivel de las Naciones Unidas presenta un informe que inspiraría un año más tarde a la Doctrina de la Responsabilidad de Proteger, donde "la seguridad se entiende como un concepto que va más allá de los intereses estatales, para hablar de seguridad humana" (Zabaleta, 2014, p. 4).

Se trata de un intento por reformular el concepto de soberanía donde la seguridad del Estado deja de ser el único objeto de protección ${ }^{11}$; ahora, la integridad de las personas constituye el valor primordial, incluso si ello implica afectar el principio de no intervención derivado de la tradición westfaliana. La soberanía, en consecuencia, es entendida como una "carga de responsabilidad que obliga a los Estados a responder del bienestar de su población” (Oficina del Asesor Especial sobre la Prevención del Genocidio, 2016).

Así, emerge una suerte de "principio de la responsabilidad de proteger" (Díaz, 2012 , pp. 3-4), como criterio orientador para el comportamiento de los Estados. Se trata de un postulado recogido en el Documento Final de la Cumbre de 2005:

Cada Estado es responsable de proteger a su población del genocidio, los crímenes de guerra, la depuración étnica y los crímenes de lesa humanidad. Esa responsabilidad conlleva la prevención de dichos crímenes, incluida la incitación a su comisión, mediante la adopción de las medidas apropiadas y necesarias. Aceptamos esa responsabilidad y convenimos en obrar en consecuencia (Asamblea General de la ONU, 2005, p. 33 ).

El Secretario General de las Naciones Unidas en sus informes de 2005 y 2009 (Asesor Especial Responsabilidad de Proteger, 2009) $)^{12}$ reivindicó los postulados y los compromisos que adquirieron los Estados a partir de la suscripción del Documento Final de la Cumbre de 2005. Aunque en ninguno de ambos informes se alude a la seguridad humana, se refieren algunos de sus aspectos más importantes como las relaciones entre derechos humanos, seguridad y desarrollo.

La responsabilidad de proteger sugiere que cualquier Estado podría ser intervenido - incluso militarmente- si no cumple con el deber de proteger a su población. Se
${ }^{11}$ Se alude puntualmente al becho de que las amenazas a la seguridad de quienes habitan el territorio de un Estado ya no le conciernen unicamente a él, sino a la comunidad internacional en su conjunto. En otraspalabras, algo que tradicionalmente se entendió propio -léase, del dominio soberano-de los Estados, como la administración de los desórdenes en su territorio - en el entendido de que otros Estados no podrian intervenir en tales asuntos-, aparece seriamente cuestionado con ocasión del concepto de seguridad humana y la doctrina de la responsabilidad de proteger.

${ }^{12}$ A partir de confusiones sobre los alcances de la responsabilidad de proteger, en 2010 se indica que la prevención es la clave en esta doctrina y que se debian fortalecer los sistemas de alerta temprana dentro de las $\mathrm{Na}$ ciones Unidas (Asesor Especial Responsabilidad de Proteger, 2010; Secretario General Naciones Unidas, 2010). Al año siguiente se enfatiza en el rol de las organizaciones regionales $y$ subregionales y se retoma el concepto de "formación de capacidades", mencionado en el Documento Final de la Cumbre de 2005, en el sentido de "ayudar a los

tersitarios 39

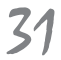


$\Rightarrow$

Estados a desarrollar su capacidad para prevenir o detectar atrocidades masivas" (Secretario General Naciones Unidas, 2011).

\section{tersitorias 39}

trata de un postulado donde la seguridad humana es presentada de manera indivisible dentro de la comunidad de Estados, aunque son solo algunos de ellos los que la han promovido, y solo algunos de ellos los que han sido intervenidos.

Así, pese a su declarada universalidad, la aplicación de esa doctrina ha planteado una división entre los Estados: por un lado, Estados desarrollados de Occidente - que pueden proveer seguridad humana a su población-, y, por otro, algunos Estados del Tercer Mundo - definidos como subdesarrollados o débiles - que no pueden hacerlo y que por ello deben ser intervenidos-(Johns, 2015). Dicha imposibilidad de que algunos Estados protejan a sus poblaciones hace que se use al desarrollo como una herramienta para garantizar la seguridad en aquellos contextos de especial conflictividad (C.A.s.E. Collective, 2006), o que se use la seguridad para garantizar mejores niveles de desarrollo en países intervenidos.

Se busca, por esa vía, evitar que las situaciones que afectan la seguridad de dichos países se "contagien" a otros. La intervención, en este sentido, busca neutralizar amenazas como el terrorismo en espacios no gobernados - como los Estados fallidos - para que no "infecten" otros que aún no están afectados por esas amenazas (Kienscherf, 2011). Las violaciones a los derechos humanos en un Estado, al ser definidas como problemas de seguridad, obligan a la comunidad internacional a intervenir para detener tales violaciones.
Aunque en algunos de los textos de Kaldor $(2008,2011)$ y de la Unidad de Seguridad Humana de las Naciones Unidas se ha intentado desligar a la seguridad humana de la responsabilidad de proteger, tales diferencias no son tan claras. El concepto de seguridad humana parece estar mucho más emparentado con la responsabilidad de proteger de lo que podría pensarse como se muestra a continuación.

\subsubsection{La seguridad humana y -algunas - violaciones de devechos bumanos como problemas de seguridad}

Como se apuntó, la formación inicial del concepto de seguridad humana se podría situar en el discurso del desarrollo humano promovido por el PNUD en 1994. Sin embargo, la seguridad humana ha sido empleada para fines distintos a los que motivaron su formulación; se trata de un concepto cuya amplitud ha permitido que de él emerjan preguntas sobre temas tan diversos como la soberanía, la intervención, el rol de las instituciones regionales y globales y las relaciones entre el Estado y sus ciudadanos (Evans, 2004).

En el contexto de la doctrina de la responsabilidad de proteger, la seguridad humana parece haber propuesto un nuevo y más amplio catálogo de "amenazas" y, si bien más adelante la ONU señala que seguridad humana y responsabilidad de proteger suponen cosas distintas, las constantes menciones a la seguridad humana cuando se hablaba de responsabilidad de proteger 
hasta el 2009 sugieren que hay más encuentros que desencuentros entre ambos conceptos.

La 'responsabilidad de proteger' se basa $[\ldots]$ en un concepto liberal desarrollado en los últimos años, el de la 'seguridad humana', que constituye la contraparte del concepto de seguridad estatal. Su premisa central es el individuo y se sirve de las categorías de la seguridad estatal como 'amenazas', 'peligros', etcétera, a fin de asegurar al individuo la ausencia de miedo y necesidad. Este concepto en su forma actual está diseñado a extender más el campo de protección del individuo y limitar más el ámbito de la soberanía estatal.

$[\ldots]$

El concepto actual de 'seguridad humana', así como lo entiende la CIISE, es sumamente ambiguo, pues incluye todo un conglomerado de atributos, prerrogativas, derechos de los individuos, incluyendo los derechos humanos, lo que complica innecesariamente el sistema normativo de la ONU ya abultado de normas y conceptos. El Documento Final ha incorporado la 'seguridad humana' solo en su aspecto de libertad de temor y miseria, y al igual que la 'responsabilidad de proteger', la tiene bajo examen, existiendo además la tarea de definirla en el futuro (Añaños, 2009, pp. 185-186).

Con las intervenciones -incluidas aquellas de tipo militar- en la responsabilidad de proteger se relativiza el principio de soberanía estatal, sobre el presupuesto de que si el Estado debe garantizar "normalmente" la protección de los derechos de sus nacionales - proveerles seguridad-, cuando esta obligación es incumplida, otros Estados tendrían el deber de intervenir con medios excepcionales para garantizar dicha protección (Watson, 2011).

Por otra parte, con la responsabilidad de proteger, como señala Kienscherf (2011), la seguridad humana asumiría su forma más extrema, como doctrina contrainsurgente donde las intervenciones humanitarias servirían para proveer seguridad, desarrollo y buen gobierno a la población local de los países intervenidos, como una forma de gestión - gobiernode la inseguridad que podría trasladarse de unos lugares a otros.

En este orden de ideas, aquel entendimiento de la seguridad humana del Informe sobre Desarrollo Humano de $1994^{13}$ parece haber variado. Asociado con la indivisibilidad e interdependencia con la que se entienden las amenazas a la seguridad de las personas - pero también debido a su vaguedad-, como apuntó Añaños (2009), termina sirviendo de fundamento a la doctrina de la responsabilidad de proteger.

Aunque la expresión 'seguridad humana' no se menciona expresamente en gran parte de los informes arriba referidos sobre responsabilidad de proteger, con excepción del informe de la Comisión Internacional sobre Intervención y Soberanía de los Estados de 2001, donde expresamente se indica que la seguridad humana es uno de los fundamentos de esa doctrina de intervención, sí se encuentran menciones a la
13 "[...] la seguridad bumana se expresa en un niño que no muere, una enfermedad que no se difunde, un empleo que no se elimina, una tensión étnica que no explota en violencia, un disidente que no es silenciado" (PNUD, 1994, p. 25).

territarias 39

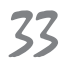


${ }^{14}$ En contra de esta postura está Hettne (2010), quien, a propósito de las relaciones entre seguridad y desarrollo, sostiene: "[...] As noted above, implied in concepts such as 'buman security', 'buman development', 'bumanitarian emergen$c y$ ' and 'bumanitarian intervention' was the idea of a transnational responsibility for human welfare: the responsibility to protect" (p. 44). En el mismo sentido, un autor como Chandler (2012) (Duffield 2010, 63; Stern \& Öjendal 2010, 17) manifiesta que hay estrechos vinculos entre responsabilidad de proteger y seguridad humana, destacando especialmente los momentos posintervención.

\section{territarios 39}

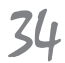

seguridad humana en los informes sobre desarrollo humano que periódicamente presentó el PNUD —en unos con más visibilidad que en otros, como ocurrió con los que se publicaron en los años 1999 y 2005 - , lo que es coherente con los orígenes de este concepto, vinculados con los debates sobre el desarrollo y no con los de seguridad (de Wilde, 2008).

Para mostrar que la aplicación del concepto de seguridad humana se refería al campo del desarrollo y no de la seguridad, tanto el Secretario General de las Naciones Unidas en algunas de sus presentaciones ante los miembros de la Asamblea General de la ONU (Secretary General-UN, 2010; Secretary-General UN, 2012), como la Asamblea General de ese organismo se han pronunciado para precisar las diferencias entre la seguridad humana y la doctrina de la responsabilidad de proteger (General Assembly-UN, 2012) ${ }^{14}$.

Ahora bien, Canadá no fue el único Estado preocupado por la seguridad internacional en el contexto de nuevas amenazas a las que se hizo mención arriba. La Unión Europea también conformó una comisión para pensar las amenazas a la seguridad internacional en el contexto de un mundo globalizado.

En 2003, y a raíz de las guerras que Estados Unidos y sus aliados habían iniciado en Afganistán e Irak como parte de una Estrategia Europea de Seguridad, se conforma un grupo de expertos liderado por la profesora Mary Kaldor -El Grupo de Estudios sobre las Capacidades de Europa-, que presenta un informe, llamado
"Informe Barcelona", en el que se insiste en que la "Unión Europea [...] tiene un interés crítico en desarrollar capacidades que le permitan realizar una contribución a la seguridad humana global" (Grupo de Estudios sobre las Capacidades de Europa 2007, p. 193).

Según el "Informe Barcelona", no son relevantes todos los contextos donde haya amenazas a la seguridad humana. Los que interesan son los de algunos países con situaciones de violencia y fragilidad institucional, de ahí que las amenazas a las que alude se refieran solo a algunos Estados.

[...] La cuestión fundamental del enfoque de seguridad humana radica en que los europeos no pueden estar seguros mientras que otros en el mundo viven en una grave situación de inseguridad. En los estados fallidos y en las regiones en conflicto, la economía criminal se extiende y se exporta: el tráfico de drogas, el tráfico de seres humanos y la facilidad con que se adquieren las armas pequeñas, e incluso la brutalización de la sociedad, no se limitan a la zona de conflicto sino que se sienten más allá de ella, inclusive en Europa. Cuando el Estado se derrumba, se movilizan las ideologías comunalistas, generalmente arraigadas en la religión o la etnicidad, y mientras que ello conduce, en primer lugar y ante todo, a una espiral de violencia dentro de las zonas en conflicto, las redes terroristas también prosperan y reclutan a sus adeptos a partir de esas situaciones (Grupo de Estudios sobre las Capacidades de Europa, 2007, p. 166). 
Esta selectividad de lo universal, como se podría llamar a la manera como se escogen algunos Estados para intervenir, ese entendimiento de lo "humano" universal en la seguridad humana, propone interrogantes como los siguientes:

- Si la preocupación por la protección de los derechos humanos es "universal", ¿por qué solo se enfoca en algunos Estados, normalmente definidos como fallidos - Kosovo, Timor Oriental, Sierra Leona-? (Grupo de Estudios sobre las Capacidades de Europa, 2007, p. 165$)$.

- ¿Acaso la generación de inseguridades básicas respecto a la libertad individual es un patrimonio exclusivo de ese tipo de Estados? ¿En los Estados “desarrollados" no hay amenazas a la seguridad humana de sus poblaciones? ¿En ellos no hay violaciones a los derechos humanos que afecten la seguridad?

Así las cosas, la referida 'selectividad' expresa la manera como en torno a ciertos valores presumiblemente valiosos y compartidos por todos los actores en el escenario internacional - como la vigencia de los derechos humanos-, en realidad opera una lógica según la cual aquello "humano" - de los derechos humanos y de la seguridad humana- resulta mucho más amenazado en unos casos que en otros, en lo que supone un aprovechamiento, o como se ha planteado en este artículo un uso político de este tipo de conceptos, para alcanzar fines que podrían ubicarse incluso en sus antípodas como cuando se pretende proteger los derechos humanos violentándolos masivamente con ocasión de las intervenciones militares.

Esta selectividad de las intervenciones, como apunta Hernández, se puede notar especialmente en el caso de Libia:

La intervención en Libia supone el primer caso en el que el Consejo de Seguridad ha aplicado la doctrina de la responsabilidad de proteger para autorizar una intervención militar con fines de protección humana, a pesar de no explicitarlo en el mandato de la Resolución. La intervención en Libia reavivó la actualidad de la [Responsabilidad de Proteger], pudiendo ser considerado el primer caso en que se ha utilizado la fuerza armada en nombre de este principio. Sin embargo, parece que su uso no ha cumplido ni con los preceptos establecidos ni con las expectativas proyectadas.

El conflicto reabre el aparentemente eterno debate sobre la intervención con fines humanitarios. A pesar de contar con la legalidad la intervención no ha gozado de la legitimidad necesaria poniendo de manifiesto la instrumentalización de Naciones Unidas por parte de determinados estados. El uso politizado de este conflicto, junto con la falta de intervención en casos similares como Siria, Bahréin o Yemen, parecen mostrar que el derecho internacional no recae sobre las grandes potencias, de manera que pueden actuar tanto de forma unilateral como utilizando a NN.UU. para legitimar su política exterior (Hernández, 2015). territarios 39

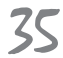


La figura 1, que expresa la selectividad de lo universal arriba enunciada, muestra cómo se han realizado intervenciones en virtud de la responsabilidad de proteger, sobre todo en operaciones de mantenimiento de paz. Como se podrá notar, solo se interviene en aquellos países a los que se define como débiles o fallidos.

Cinco años después de la publicación de la Estrategia Europea de Seguridad - en 2008-, el Consejo de la Unión Europea da a conocer el "Informe sobre la aplicación de la estrategia europea de seguridad". Se trata de un documento que alude expresamente a "responsabilidad de proteger" y la "responsabilidad compartida".

En muchos casos, los conflictos están vinculados a la fragilidad de los Estados. Países como Somalia se ven encerrados en un círculo vicioso de gobiernos débiles y conflictos recurrentes. Hemos procurado romper este círculo, combinando la ayuda al desarrollo con medidas destinadas a mejorar la seguridad. Los aspectos de reforma del sector de la seguridad y de desarme, desmovilización y reintegración, son un factor clave de la estabilización y la reconstrucción tras un conflicto, y han constituido el eje de nuestras misiones en Guinea-Bissau y en la República Democrática del Congo [...] (Consejo de la Unión Europea, 2009, p. 19).

En este sentido, se trata de una articulación de la seguridad humana a fases posteriores a las intervenciones, con el fin de proteger y empoderar poblaciones vulnerables, como ocurrió en Lybia, donde la intervención de 2011 habría significado una reorientación de la responsabilidad de proteger hacia la emancipación y la agencia de su población (Chandler, 2012), aunque como se muestra en casos como los de Cambodia y Myanmar, solo reprodujo

Figura 1. Operaciones del Mantenimiento de Paz de las Naciones Unidas

\section{tersitarias 39}

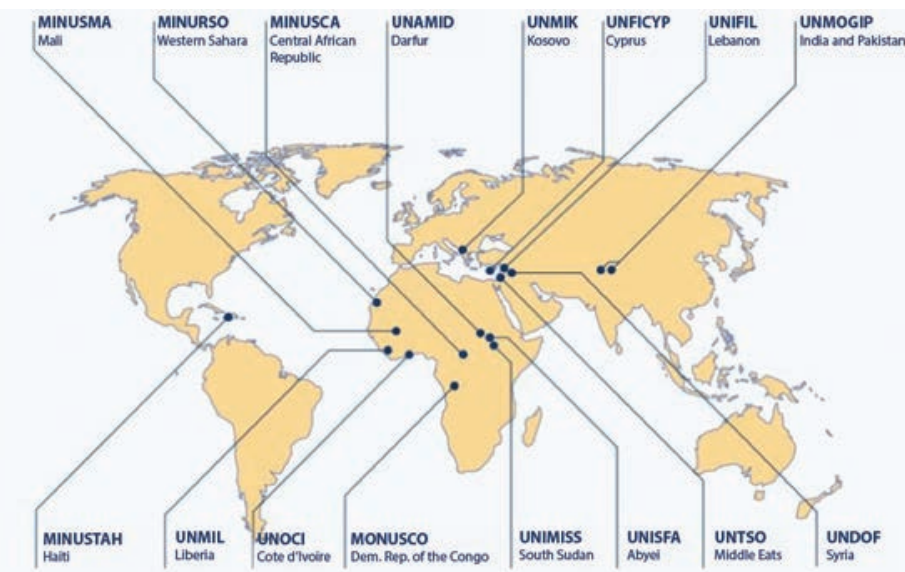

Fuente: tomado de Hernández, 2015. 
viejas prácticas autoritarias de tipo estadocéntrico, ante la ausencia de un discurso y un diseño institucional que permitieran abordar ciertos asuntos como problemas de seguridad humana para proteger a la población (Jacob, 2014).

Así, la seguridad humana, como fundamento de la doctrina de la responsabilidad de proteger, hizo posible construir como problemas de seguridad internacional la violación de derechos humanos en algunos países. Las respuestas que se diseñaron incluyeron las intervenciones —incluidas las de tipo militar-. Solo las afectaciones a los derechos humanos generadas en los Estados definidos como subdesarrollados, débiles o fallidos constituyen amenazas a la seguridad internacional.

\subsection{El subdesarrollo de algunos países como problema de seguridad}

El proceso de securitización que se menciona en este apartado se refiere a los usos políticos del concepto de seguridad humana por instituciones de las Naciones Unidas, como la Unidad de Seguridad Humana de las Naciones Unidas y el Fondo Fiduciario para la Seguridad Humana, creados como resultado de las recomendaciones de la Comisión sobre Seguridad Humana (Comission on Human Security, 2003; UN-Human Security Unit, 2016a). La securitización del subdesarrollo evoca el nexo entre seguridad y desarrollo o cómo por medio de estrategias de desarrollo se puede garantizar la seguridad en determinados contextos.
Cabe recordar, no obstante, que los vínculos entre seguridad y desarrollo - es decir, las relaciones entre sus ideas, procesos u objetos (Stern \& Öjendal, 2010) anteceden a la seguridad humana. Ya en los tiempos de la Guerra Fría y de la Doctrina de la Seguridad Nacional había un fuerte vínculo entre ambas nociones (Duffield, 2007).

La relación entre seguridad y desarrollo, según la perspectiva de Duffield (2010) se funda en la idea de que, por medio del desarrollo, se puede alcanzar la seguridad internacional. En este sentido, situaciones de pobreza y desigualdad pueden generar contextos de inseguridad que en el actual mundo globalizado e interconectado pueden extenderse más allá de las fronteras de los Estados donde se originaron, con lo cual "la seguridad humana necesita el desarrollo humano" (Glasius, 2008, p. 41), o, según el Secretario General de la ONU (citado por Stern \& Öjendal, 2010) "un mundo más seguro sólo es posible si los países pobres tienen una oportunidad real para desarrollarse" (p. 5).

Así las cosas, el nexo entre seguridad y desarrollo hace de este una herramienta para garantizar aquella. Al promover el concepto de seguridad humana, se trató de desarrollar las "potencialidades (a las que Nussbaum llama 'capacidades básicas')" (Marhia, 2013, p. 23) para que la vida sea completamente humana, lo que según Marhia (2013) hace que "algunos humanos son más humanos que otros" (p. 23).

Según el informe Human Security Now, publicado gracias a la iniciativa del territarias 39 
gobierno japonés, la promoción de la seguridad humana debe estar atada a la protección y al empoderamiento (Comission on Human Security, 2003; Nishikawa, 2009), $\mathrm{y}$, en consecuencia, las nuevas instituciones que se crean en las Naciones Unidas - entre ellas el Fondo Fiduciario para la Seguridad Humana y la Unidad creada para su administración- buscaron "mejorar la vida y el sustento de las personas que se enfrentan a situaciones complejas y multidimensionales de inseguridad humana", por medio de "herramientas para aplicar el enfoque de la seguridad humana en programas y proyectos" (ONU-Human Security Unit, 2016).

Una revisión del conjunto de los materiales disponibles en el sitio web de la Unidad de Seguridad Humana de las Naciones Unidas muestra que, a diferencia de lo que ocurría en los tiempos de la Guerra Fría, cuando los programas de desarrollo buscaban "quitarle el agua al pez" para contener la amenaza comunista en virtud de la Doctrina de la Seguridad Nacional (Calderón, 1999; Rueda, 2000), ahora con el concepto de seguridad humana, los programas de desarrollo buscan la protección y el empoderamiento.

Se trataría de completar lo que está incompleto en países con problemas de desarrollo: "podría ser falta de educación, falta de capacidad, la incapacidad para ahorrar correctamente o la necesidad de una mayor conciencia de género, estas son las ausencias que significan que la vida no se puede vivir adecuadamente" (Duffield, 2010, p. 61). O, como sugirió el Grupo
Japonés de Estudios Afganos en 2008 (citado por Hynek, 2012a), “mejorar la seguridad humana en las comunidades [...] [locales] para que sean resistentes al terrorismo es la forma más eficaz de enfrentarlo" (p. 125).

El material dispuesto en la página web de la Unidad de Seguridad Humana (http://www.un.org/humansecurity/) incluye 53 archivos que se dividen en: (i) Boletines, (ii) Debates en la Asamblea General de las Naciones Unidas, (iii) Informes presentados por el Secretario General, (iv) Materiales de difusión y (v) Herramientas de Seguridad Humana. De la lectura del material mencionado se puede inferir que:

- Los usos políticos de la seguridad humana en los programas financiados por el Fondo Fiduciario de Seguridad Humana, de la Unidad de Seguridad Humana, están articulados a programas de desarrollo y construcción de paz, pero también han implicado proyectos sobre cambio climático, violencia urbana, reducción de la pobreza y salud (Human Security Research Project, 2013);

- Diversas instancias deciden pronunciarse sobre la relación entre responsabilidad de proteger y seguridad humana, llegando a plantear que son nociones distintas en los niveles conceptual y de su implementación. Hay casi una obsesión en distintos documentos por afirmar, recordar, recalcar que la seguridad humana es distinta a la responsabilidad de proteger;

Julián ANDrés MuÑoz TejadA 
- Dicha disputa por el significado, como se podría llamar a la tensión por definir los límites entre ambas nociones, pone de presente que, aunque hoy tengan desarrollos propios y cuenten con instituciones especializadas, no niega que en un primer momento ambos conceptos estuvieron íntimamente ligados.

En resumen, la Unidad de Seguridad Humana, adscrita al Sistema de las $\mathrm{Na-}$ ciones Unidas, entendió que las guerras en países pobres y su escaso desarrollo debían ser asumidos como problemas de seguridad. Para hacerle frente a las amenazas que se pudieran generar en tales contextos, recurrió a estrategias como los programas financiados por el Fondo Fiduciario para la Seguridad Humana de las Naciones Unidas.

Como ocurrió con la doctrina de la responsabilidad de proteger, los programas de desarrollo también son aplicados selectivamente, como se puede apreciar en la siguiente imagen que muestra en color naranja los Estados donde se han ejecutado programas de desarrollo financiados por la Unidad de Seguridad Humana. La figura 2 muestra cómo los programas de desarrollo de la Unidad de Seguridad Humana de las Naciones Unidas se concentran en países de llamado "tercer mundo" o "subdesarrollados".

Usada de esa manera, la seguridad humana sirvió para hacer del subdesarrollo de algunos países un problema de seguridad global. Así pensada, la securitización del desarrollo se articula al discurso hegemónico fundado en la universalidad de "lo humano" como un estado de cosas - de desarrollo- deseable y replicable en todos los países. De nuevo, cabría preguntar, ¿quién no estaría de acuerdo con que países pobres se desarrollen? El problema de fondo a este interrogante, como bien lo ha señalado Duffield (2005), es que detrás de todo ese apoyo de la comunidad internacional subyace un ejercicio de control

Figura 2. Países donde se han ejecutado programas de desarrollo financiados por la Unidad de Seguridad Humana

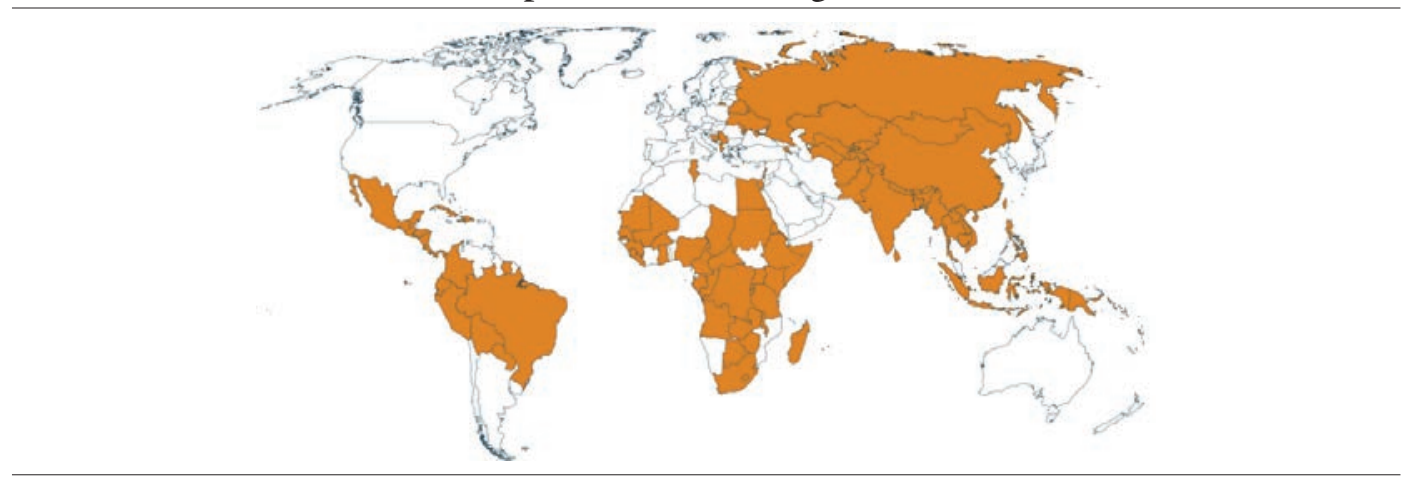

Fuente: Human Security Unit (2016, p. 10). 
cada vez más asfixiante para los Estados que son "beneficiados" con los apoyos de desarrollo de la comunidad internacional.

A partir del nexo entre seguridad y desarrollo al que se refiere Duffield, es posible advertir que detrás de los programas de desarrollo hay un interés por controlar a los países subdesarrollados, una suerte de control biopolítico, que en sus palabras posibilita ejercicios de control sobre poblaciones del sur por parte de agencias internacionales. Entiende este autor que la seguridad humana es un principio de formación de "humanos", esto es de poblaciones productivas y sobre todo de gestión de inseguridades derivadas del subdesarrollo (Duffield, 2005).

No hay, por lo tanto, una ruptura efectiva en las prácticas de control de unos Estados sobre otros, si se compara, por ejemplo, la seguridad humana con la Doctrina de la Seguridad Nacional, aunque esta última aluda a enemigos internos y la primera no. Cambia la forma de adjetivar la seguridad - ya no seguridad nacional, sino seguridad humana- pero es difícil identificar cambios concretos en la manera como unos Estados poderosos siguen incidiendo en otros que no lo son.

\section{Apuntes finales}

El concepto de seguridad humana emerge en el contexto de la pos-Guerra Fría y posibilitó la ampliación del campo discursivo de la seguridad. Según esta forma de pensar la seguridad, el Estado no era el único objeto

\section{tersitarias 39}

ser asumidas como uno de tales objetos de protección. Por otra parte, el concepto de seguridad humana, al plantear que la seguridad se puede afectar desde una de múltiples dimensiones, amplió también el tipo de amenazas desde las que se puede afectar la seguridad.

En este contexto de ampliación del campo discursivo de la seguridad, el concepto de seguridad humana tuvo significados diversos. En torno a él se construyeron al menos dos discursos de seguridad humana. En el primero, la seguridad humana critica tradicionales visiones militaristas sobre la seguridad; en el segundo, al ser usado por Estados poderosos constituyó una nueva ortodoxia. De la revisión de los dos procesos de securitización de este último discurso se puede concluir que:

En uno de ellos, la seguridad humana se usó como fundamento de la doctrina de la responsabilidad de proteger, en virtud de la cual se plantea posibilidad de que unos Estados intervengan en otros con el propósito de proteger los derechos humanos de los ciudadanos amenazados por la acción u omisión del Estado intervenido.

La dificultad para diferenciar los conceptos de seguridad humana y responsabilidad de proteger, como se mostró aquí de la mano de diversos autores, además de las coincidencias en los fundamentos y propósitos de ambos, muestra que, pese al interés de las Naciones Unidas por precisar sus límites, y al hecho de que hoy existe mayor claridad sobre las especificidades de cada uno de ellos, no niega que en sus inicios la seguridad humana 
inspiró o fundamentó la responsabilidad de proteger.

En el otro proceso, la seguridad humana se usó en programas de desarrollo liderados por las Naciones Unidas para hacer del subdesarrollo de algunos países un problema de seguridad internacional. Se piensa desde esta perspectiva que el incremento de las capacidades de los Estados y sus ciudadanos, por medio de programas de desarrollo en países que padecen conflictos armados, contextos de violencia crónica, o que están amenazados por desastres naturales, puede ser una herramienta para garantizar su seguridad y evitar que los brotes de inseguridad se extiendan y afecten otros países.

Según Duffield (2005), hay puntos de encuentro entre la Guerra Fría y la "posGuerra Fría” en la que emerge la seguridad humana. Particularmente, habría un desplazamiento en las amenazas que ya no están representadas en el comunismo internacional, sino en el terrorismo. En este sentido, como se apuntó, el centro de las preocupaciones del desarrollo humano y la seguridad humana se focaliza en poblaciones cuya inseguridad podría "contagiar" a países desarrollados.

En conclusión, los usos políticos del concepto de seguridad humana muestran que "lo humano", aquel universal al que apela la seguridad humana en el escenario internacional, dibuja amenazas o peligros comunes a toda la comunidad internacional. Un análisis de sus usos políticos, como el que aquí se efectuó, muestra cómo el concepto de seguridad humana fue usado como sustento de otras formas de exclusión y de dominación de unos Estados sobre otros.

En suma, los dos usos políticos de la seguridad humana aquí explorados muestran cómo el sentido o significado de un concepto está ligado al aprovechamiento que hagan de él algunos actores en el campo político, y que, tal como apuntó Williams (2008), la seguridad es ante todo una poderosa herramienta política que permite priorizar algunos problemas sobre otros.

Cabe precisar, no obstante, que la "culpa" no es de la seguridad, - sea esta nacional, ciudadana, o humana-, sino de la manera como se le instrumentaliza. Entendida como instrumento, la seguridad también puede ser usada para alcanzar fines distintos a los de control que se sugirieron en este artículo. Tal es el caso del Observatorio de Seguridad Humana de Medellín, que en sus estudios ha mostrado cómo no siempre que se demanda la provisión de seguridad se está haciendo un llamado a medidas excepcionales o a respuestas de tipo violento.

En una posterior publicación se mostrará cómo este Observatorio y las comunidades que estudió en Medellín usaron el concepto de seguridad humana para demandar mejoras en su bienestar y en el ejercicio de sus derechos, en lo que denominaron una "seguridad humana desde abajo". territarias 39

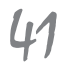




\section{Referencias}

Acharya, A. (2004). A Holistic Paradigm. Security Dialogue, 35(3), 355-356.

Acharya, A. (2016). Idea Shift: How Ideas From the Rest are Reshaping Global Order. Third World Quarterly, 37(7), 1156-1170.

Añaños, M. C. (2009). La "responsabilidad de proteger" en Naciones Unidas y la Doctrina de la "Responsabilidad de proteger". UNISCI Discussion Papers 21, 164-192.

Asamblea General (ONU). (2005). Resolución 16 de septiembre de 2005. Documento Final de la Cumbre 2005.

Asamblea General (ONU). (2009). Resolución 63 aprobada por la Asamblea General el 14 de septiembre de 2009.

Asesor Especial Responsabilidad de Proteger. (23 July 2009). Observaciones a la Asamblea General sobre la Responsabilidad de Proteger. New York: Organicación de las Naciones Unidas.

Asesor Especial Responsabilidad de Proteger. (2010). Informal interactive Dialogue on Early Warning, Assesment and the Responsibility to Protect, 9 angusto 2010. New York: United Nations.

Bajpai, K. (2004). An Expression of Trheats Versus Capabilities Across Time and Space. Security Dialogue, 35(3), 360361.

Balzacq, T. (2011). Enquiries into methods. En T. Balzaq (autor), Securitization Theory. How security problems emerge

\section{territarias 39}

42 Routledge.
Balzacq, T., \& Guzzini, S. (2015). Introduction: 'What kind of theory - if any - is securitization?'. International Relations, 29(1), 97-102.

Bourdieu, P. (1990). Sociología y cultura. México: Grijalbo.

Buzan, B., Wrever, O., \& de Wilde, J. (1999). Security. A new Framework for Analysis. Colorado: Reiner.

C.A.S.E. Collective. (2006). Critical Approaches to Security in Europe: A Networked Manifesto. Security Dialogue, 37(4), 443-487.

Calderón, O. (1999). La seguridad estatal en Colombia. América Latina Hoy 23, 91-103.

Chandler, D. (2012) Resilience and human security: The post-interventionism paradigm. Security Dialogue, 43(3), 213219.

Comisión Internacional sobre Intervención y Soberanía de los Estados (CIISE). (2001). La responsabilidad de proteger. Informe de la Comisión Internacional sobre Intervención y Soberanía de los Estados.

Comission on Human Security (2003). Human Security Now. New York: Comission on Human Security.

Consejo de la Unión Europea (2009). Una Europa segura en un mundo mejor. En Estrategia Europea de Seguridad. Una Europa segura en un mundo mejor (pp. 27-43). Luxemburgo: Oficina de Publicaciones de la Unión Europea.

Christie, R. (2010). Critical voices and Human Security: to endure, to engage or 
to critique? Security Dialogue, 41(2), 169-190.

De Certeau, M. (2007). La invención de lo cotidiano 1 , artes de hacer. México: Universidad Iberoamericana.

De Wilde, J. (2008). Speaking or Doing Human security. En M. den Boer, \& J. de Wilde (eds.), The viability of human security (pp. 225-254). Amsterdam: Amsterdam University Press.

Díaz, C. (2012). La responsabilidad de proteger en el derecho internacional contemporáneo: entre lo conceptual y la práctica internacional. Revista Elecrónica de Estudios Internacionales, 24, 1-40.

Duffield, M. (2005). Human Security: Linking Development and Security in an Age of Terror. Paper prepared for the GDI panel "New Interfaces between Security and Development", $11^{\circ}$ General Conference of the EADI, 21-24 september. Bonn.

Duffield, M. (2007). Development, securty and unending war. Governing the world of peoples. Cambrigde: Polity Press.

Duffield, M. (2010). The Liberal Way of Development and the DevelopmentSecurity Impasse: Exploring the Global LIfe-Chance Divide. Security Dialogue, 41(1), 53-76.

Evans, P. (2004). A Concept Still on the Margins, but Evolving from Its Asian Roots. Security Dialogue, 35(3), 363-364.

Ferreira, T. (2013). Human Security Paradigms and Economic Crisis in first year of the North Caucasus Federal District. Brazilian Political Science Review 7, 74-93.
Gallardo, B. (2014). Usos politicos del lenguaje. Un discurso paradójico. Barcelona: Anthropos Editorial.

García, C. (2007). Seguridad humana y política exterior japonesa: contexto, concepto y aplicación. CIDOB d'Affers, 76, 79-95.

Gasper, D. (2015). Human Security Thinking in Practice "Personal Security", "Citicen Security", Comprehensive Mappings. Contemporary Politics, 21(1), 1-24.

Glasius, M. (2008). Human Security from Paradigm Shift to Operationalization: Job Description for a Human Security Worker. Security Dialogue, 39(1), 31-54.

Grupo de Alto Nivel sobre las Amenazas, los Desafíos y el Cambio (2004). Un mundo más seguro: la responsabilidad que compartimos. Informe del Grupo de alto nivel sobre las amenazas, los desafíos y el cambio.

Grupo de Estudios sobre las Capacidades de Europa (2004). Informe Barcelona. Barcelona.

Grupo de Estudios sobre las Capacidades de Europa (2007). Una doctrina de seguridad humana para Europa. Informe Barcelona. Revista CIDOB D'AFERS, 76, 159-199.

Hernández, M. (12 de Marzo de 2015). La Responsabilidad de Proteger. Recuperado de http://elordenmundial. com/2015/03/12/la-responsabilidad-de-proteger/ territarias 39

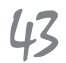


Hettne, B. (2010). Development and Security: Origins and Future. Security Dialogue, 41(1), 31-52.

Hynek, N. (2012). Human security as statecraft. Structural conditions, articulations and unintended consequences. New York: Routledge.

Hynek, N. (2012a). The domopolitics of Japanese human security. Security Dialogue, 43(2), 119-137.

Jacob, C. (2014). Practising civilian protection: Human security in Myanmar and Cambodia. Security Dialogue, 45(4), 392-408.

Johns, L. (2015). A critical evaluation of the concept of human security. Recuperado de http://www.e-ir. info/2014/07/05/a-critical-evaluation-of-the-concept-of-human-security/

Kaldor, M. (2011). El poder y la fuerza. La seguridad de la población civil en un mundo global. México: Tusquets Editores.

Kaldor, M., Martin, M., \& Selchow, S. (febrero de 2008). Human Security: A European Strategic Narrative. International Policy Affairs, 1-4.

Kamal, M. (2007). Hegemony, Perilous Empire and Human Security. En J. Camilleri, L. Marshall, M. Michael, \& M. Seigel (eds.), Asia-Pacific Geopolitics. Hegemony vs. Human Security (pp. 23-74). Cornwall, Reino Unido: MPG Books.

Kienscherf, M. (2011). A programme of global pacification: US counterinsur-

\section{territarias 39} 44 human (in)security. Security Dialogue, 42(5), 517-533.

Laclau, E. (1996). Emancipación y diferencia. Buenos Aires: Ariel.

Laclau, E. (2004). Discurso. Topos \& Tropos $1,1-7$.

Macfarlane, N. (2004). A Useful Concept that Risks Losing Its Political Salience. Security Dialogue, 35(3), 368-369.

Magallón, C. (2007). Seguridad bumana y lucha antiterrorista. El impacto sobre el desarrollo. Recuperado de http://www. seipaz.org/documentos/1MagallonSe guridadhumana.pdf

Marhia, N. (2013). Some humans are more Human than Others: Troubling the 'human' in human security from a feminist perspective. Security Dialogue, 44(1), 19-35.

Mendia, I., \& Saillard, D. (2013). Feminismo y Seguridad Humana: Encuentros y Desencuentros. En K. Pérez de Armiño, \& M. Irantzu, Seguridad Humana. Aportes Criticos al Debate Teórico y Político (pp. 227-246). Madrid: Tecnos.

Morillas, P. (2007). Génesis y evolución de la expresión seguridad humana. Un repaso histórico. Revista CIDOB d'Afers Internacionals, 47-58.

Navarrete, Z. (2009). Eclecticismo teórico en las ciencias sociales. El caso del Análisis Político del Discurso. En R. Soriano, \& M. Ávalos (coords.), Análisis Politico del Discurso. Dispositivos intelectuales en la investigación social (pp. 139-151). México: Juan Pablos Editor.

Oficina del Asesor Especial sobre la Prevención del Genocidio (2016). Respon- 
sabilidad de proteger. Recuperado de http://www.un.org/es/preventgenocide/adviser/responsibility.shtml

ONU-Human Security Unit. (2016). About Human Security Unit. Recuperado de http://www.un.org/humansecurity/

Pérez de Armiño, K. (2007). El concepto y uso de la seguridad humana: análisis crítico de sus riesgos y potencialidades. CIDOB d'Afers Internacionals, 76, 5977.

Pérez de Armiño, K. (2013). Seguridad humana y estudios críticos de seguridad: de la cooptación a la emancipación. En K. Pérez de Armiño, \& I. Mendia (eds.), Seguridad Humana. Aportes críticos al debate teórico y politico (pp. 23-57). Madrid: Tecnos.

PNUD (1994). Informe sobre Desarrollo Humano 1994. Un Programa para la Cumbre Mundial sobre Desarrollo Social. Nueva York: Programa de las Naciones Unidas para el Desarrollo.

PNUD (1999). Informe sobre desarrollo bumano. La mundialización con rostro humano. Nueva York: Programan de las Naciones Unidas para el Desarrollo.

PNUD (2005). Informe sobre desarrollo humano 2005. La cooperación internacional ante una encrucijada: Ayuda al desarrollo, comercio y seguridad en un mundo desigual. Nueva York: Programa de las Naciones Unidas para el Desarrollo.

Rodríguez, J. (2005). La noción de "seguridad humana": sus virtudes y sus peligros. Polis 11, 1-16.
Roznai, Y. (2014). The insecurity of human security. Wisconsin International law journal, 32(1), 95-141.

Rueda, R. (2000). De la guardia de fronteras a la contrainsurgencia. Elementos de la evolución politica e institucional del ejército colombiano 1958-1965. Bogotá: Instituto Colombiano para el Fomento de la Educación Superior (ICFES).

Saur, D. (2017). La disyunción laclausiana: entre el horizonte discursivo y el callejón begemónico (inédito).

Schering, C., \& Wood, J. (2011). Pensar la seguridad. Barcelona: Gedisa.

Secretario General Naciones Unidas (2005). Un concepto más amplio de libertad: desarrollo, seguridad y derechos humanos para todos. Informe del Secretario General, 21 de marzo de 2005.

Secretario General Naciones Unidas (2009). Hacer efectiva la responsabilidad de proteger, Informe del Secretario General, 12 de enero.

Secretario General Naciones Unidas (2010). Alerta temprana y evaluación y la responsabilidad de proteger. Informe del Secretario General, 14 de julio de 2010. Secretario General Naciones Unidas (28 de junio de 2011). La función de los mecanismos regionales y subregionales para hacer efectiva la responsabilidad de proteger. Informe del Secretario General.

Secretary General-un. (8 de marzo de 2010). Human Security. Report of the Secretary General.

Secretary General-UN (2012). Follow-up to General Assembly Resolution 64/291 on buman security. New York: UN. territarios 39

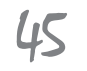


Stern, M., \& Öjendal, J. (2010). Mapping the Security-Development Nexus: Conflict, Complexity, Cacophony, Convergence? Security Dialogue, 41(1), 5-30.

Thede, N. (2008). Human Security, democracy, and development in the Americas: The Washington consensus redux? Canadian Journal of Latin American and Caribbean Studies, 33(65), 33-56.

uN-Human Security Unit (2016). About Human Security Unit. Recuperado de http://www.un.org/humansecurity/.

UN-Human Security Unit (mayo de 2016). Human Security-Trajectory and Aplication at the United Nations. Recuperado de http://www.un.org/humansecurity/sites/www.un.org.humansecurity/ files/untfhs_ppp_-_human_security_ may_2016.pdf
Watson, S. (2011). The 'human' as referent objetc? Humanitarism as securitization? Security Dialogue, 42(1), 3-20.

Williams, P. (2008). Security Studies. An introduction. En P. Williams (ed.), Security Studies. An introduction (pp. 1-12). New York: Routledge.

Wittgenstein, L. (1999). Investigaciones filosóficas. Barcelona: Ediciones: Atalaya.

Wittgenstein, L. (2011). El libro azul. Caracas: bid\&co

Zabaleta, B. (2014). Naciones Unidas y la Responsabilidad de Proteger: ¿Qué papel debe desempeñar la ONU en los conflictos internos. Documento Opinión, 58, 1-20. Recuperado de http:// www.ieee.es/Galerias/fichero/docs_ opinion/2014/DIEEEO58-2014_ NNUU_ConflictosInternos_BertaZabaletav.pdf 